\title{
The upper-hemifield advantage for masked face processing: Not just an attentional bias
}

\author{
Genevieve L. Quek ${ }^{1,2,3,4}$ - Matthew Finkbeiner ${ }^{1,2,3}$
}

Published online: 30 October 2015

(C) The Psychonomic Society, Inc. 2015

\begin{abstract}
Recent evidence suggests that face processing may be more robust in the upper visual field (UVF) than in the lower visual field (LVF). We asked whether this UVF advantage is due to an upward bias in participants' visuospatial attention. Participants classified the sex of a UVF or LVF target face that was preceded by a congruent or incongruent masked prime face. We manipulated spatial attention within subjects by varying the predictability of target location across sessions (UVF:LVF ratio of 50:50 on Day 1 and 20:80 on Day 2). When target location was unpredictable, priming emerged earlier in the UVF ( $\sim 165 \mathrm{~ms})$ than the LVF ( 195 ms). This UVF advantage was reversed when targets were more likely to be presented in the LVF. Here priming arose earlier for LVF targets ( $\sim 53 \mathrm{~ms})$ than UVF targets ( $\sim 165 \mathrm{~ms})$. Critically, however, UVF primes were processed to the same degree regardless of whether spatial attention was diffuse (Day 1) or deployed elsewhere (Day 2). We conclude that, while voluntarily directed spatial attention is sufficient to modulate the processing of masked faces in the LVF, it is not sufficient to explain the UVF advantage for masked face processing.
\end{abstract}

Genevieve L. Quek

genevieve.lauren.quek@gmail.com

1 Department of Cognitive Science, Macquarie University, North Ryde, Sydney, NSW, Australia

2 ARC Centre of Excellence in Cognition and its Disorders (CCD), Macquarie University, Sydney, NSW, Australia

3 Perception and Action Research Centre (PARC), Macquarie University, Australian Hearing Hub, North Ryde, Sydney, NSW, Australia

4 Present address: Université catholique de Louvain (UCL), 10, Place du Cardinal Mercier, 1348 Louvain-La-Neuve, Belgium
Keywords Attention · Faces · Vertical asymmetry · Upper visual field $\cdot$ Lower visual field

\section{Introduction}

Human visual perception is not uniform across the retinal field, but, rather, is characterized by perceptual asymmetries arising from the brain's preferential response to particular stimulus types at different retinal locations. Investigations of vertical asymmetry in visual perception have historically favored the lower visual hemifield relative to the upper visual hemifield. For example, spatial resolution (Talgar \& Carrasco, 2002) and contrast sensitivity (Carrasco, Penpeci-Talgar, \& Cameron, 2001; Skrandies, 1987) are enhanced in the lower visual field (LVF) compared to the upper visual field (UVF), with this asymmetry being most pronounced at the vertical meridian (Carrasco et al., 2001; Talgar \& Carrasco, 2002). Performance is also typically better in the LVF on tasks of hue discrimination (Levine \& McAnany, 2005), perception of illusory contours (Rubin, Nakayama, \& Shapley, 1996), motion processing (Amenedo, Pazo-Alvarez, \& Cadaveira, 2007; Edwards \& Badcock, 1993; Levine \& McAnany, 2005), global processing (Christman, 1993), and perception of coordinate spatial relationships (Niebauer \& Christman, 1998). There are select tasks which exhibit a UVF advantage, however, including visual search (Chaiken, Corbin, \& Volkmann, 1962; Fecteau, Enns, \& Kingstone, 2000; Previc \& Blume, 1993; Previc \& Naegele, 2001; Yund, Efron, \& Nichols, 1990), local processing of hierarchical stimuli (Christman, 1993), perception of apparent distance (Levine \& McAnany, 2005), and categorical judgments of position (Niebauer \& Christman, 1998). 
While vertical visual asymmetries are well documented for a range of low-level perceptual stimulus types, it is only recently that researchers have extended this line of enquiry to include higher level visual representation. Kravitz, Kriegeskorte, and Baker (2010) used functional magnetic resonance imaging (fMRI) to show that object representation in several object selective regions is constrained by vertical position. Critically for the present case, they observed a UVF bias for object representation in the left and right posterior fusiform areas, in that split-half correlations (Haxby et al. 2001) for these regions were strongest for object line drawings presented in the upper hemifield. Consistent with this report, there is now a burgeoning body of work which suggests the processing of faces - arguably one of the most important types of object we encounter in the visual world - may enjoy an upper-hemifield advantage. For example, Felisberti and McDermott (2013) have reported that participants are able to recognize previously seen faces better when those faces are initially encoded in the upper hemifield rather than the lower hemifield. Within the neurophysiological literature, Liu and Ioannides (2010) have shown that magnetoencephalography (MEG) peaks elicited by emotional faces in regions such as the medial prefrontal cortex (MPFC), left fusiform face area (FFA), and left occipital face area (OFA) arise earlier for faces presented in the UVF compared to the LVF. Most recently, we used masked priming to show that that the UVF exhibits a clear behavioral advantage for face-sex categorization (Quek \& Finkbeiner, 2014a). Participants in our study categorized the sex of a target face by reaching to either the left or right edge of a computer monitor. The target face could appear in either the UVF or LVF and was preceded by a nonconscious masked prime face of either the same or the opposite sex (i.e., congruent or incongruent). Despite being outside participants' conscious awareness, these masked prime faces nevertheless exerted a strong influence on participants' classification of the target faces, in that the participants reached towards the correct response panel faster when the prime and target were associated with the same sex-categorization response (i.e., the congruent condition). Importantly, we found that this index of nonconscious face processing (termed the masked congruence effect, or MCE) arose earlier in time when the prime and target faces appeared in the UVF compared to the LVF. Moreover, we observed a differential benefit of attentional cueing between the vertical hemifields. Where the MCE elicited by faces in the lower hemifield depended on a valid spatial cue (i.e., no priming without spatial attention), the emergence of the MCE in the upper hemifield was unaffected by spatial cueing, suggesting that masked prime faces presented above-fixation were processed irrespective of the spatial attention's locus (see Finkbeiner \& Palermo, 2009 for a similar finding with masked UVF primes).
Although not all studies of face perception across the visual field have observed stable biases across individuals (Afraz, Pashkam, \& Cavanagh, 2010), on balance the evidence suggests that the visual system is able to process face information more efficiently at locations above fixation compared to below. What is not yet established, however, is why this might be the case. A possible explanation that we explore in the present paper is that the upper-hemifield advantage in face processing arises due to an upward bias in voluntarily directed spatial attention. We are not the first to appeal to the tendency to divide attention unevenly across the visual field as a potential explanation for vertical asymmetry in visual perception. Rezec and Dobkins (2004) proposed that an "attentional weighting" favoring the lower hemifield might explain the LVF advantage they observed in motion and orientation discrimination. Interestingly, however, there is some suggestion that biases in spatial attention may depend on stimulus type. For example, studies involving vertical line bisection (Bradshaw, Nettleton, Nathan, \& Wilson, 1985; Drain \& Reuter-Lorenz, 1996; van Vugt, Fransen, Creten, \& Paquier, 2000), object matching (Chambers, McBeath, Schiano, \& Metz, 1999), and mental scene representation (Drummond \& Tlauka, 2012) suggest an upward bias in spatial attention. Importantly for the present case, there are good reasons to think participants may be particularly prone to favoring the UVF in the context of face perception, as this region of space often carries important face information during real-world interactions. We tend to encounter human faces more frequently in the UVF, as this region corresponds to extrapersonal space (Previc, 1990). Eye gaze, a potent source of social information which guides adaptive behavior (Hood, Willen, \& Driver, 1998; Langton, Watt, \& Bruce, 2000), is also typically contained within the top half of our visual field. Thus, it is reasonable to think that participants in our previous study might have voluntarily attended to the upper hemifield even while maintaining central fixation, resulting in both the earlier emergence of priming in the (attended) UVF, and the lack of an exogenous cueing effect in this region (Quek \& Finkbeiner 2014a).

If an upward bias in voluntarily directed spatial attention does indeed explain the UVF face processing advantage, then manipulating participants' endogenously oriented spatial attention away from the UVF (i.e., towards the LVF) should attenuate the UVF advantage evident in the timecourse of the MCE for faces. To test this hypothesis, we adapted our previous design known to yield both robust masked priming effects and a clear UVF advantage for face-sex categorization (Quek \& Finkbeiner 2014a). We manipulated participants' endogenous attention to the vertical hemifields by varying the probability of UVF and LVF targets across days. On Day 1, the ratio of UVF targets to LVF targets was 50:50, such that target location was entirely unpredictable for participants. In contrast, on Day 2, targets appeared in the LVF on $80 \%$ of 
trials (20:80 UVF to LVF ratio). We reasoned that participants would be sensitive to this increased probability of LVF targets and would consequently direct their spatial attention towards the lower hemifield after a period of learning. Our predictions were as follows: For Day 1 (50:50 target location ratio), we expected the MCE would be superior for UVF prime-target pairs than for LVF prime-target pairs, replicating the UVF advantage for face-sex categorization we have shown previously (Quek \& Finkbeiner 2014a). For Day 2 (20:80 target location ratio), we predicted that masked face processing below fixation would be facilitated by participants voluntarily directing spatial attention towards this hemifield. That is, we expected the priming effect for the LVF would be equivalent to, or perhaps even superior to, the UVF priming effect from the same day. The question of critical interest was what impact our manipulation of endogenous attention on Day 2 would have on the priming effect for the $U V F$. If the documented UVF advantage for subliminal face processing should be attributed to an upward bias in participants' spatial attention, then the MCE elicited by UVF faces should suffer when participants direct their spatial attention to a different location. That is, in the measure to which the UVF advantage is due to focused spatial attention, then the redeployment of that focused spatial attention to the LVF should yield both increased performance in the LVF and a cost to the priming effect in the UVF.

Before undertaking this planned design, however, we wanted to first take account of the fact that our manipulation of target location probability across days would not allow us to counterbalance the order of conditions across participants. All participants would need to complete the conditions in a fixed order (50:50 on Day 1, 20:80 on Day 2), to ensure that the location probability learned during the 20:80 condition would not carry over and influence participants' approach to the 50:50 version of the task. Consequently, we are faced with the possibility of an artefact in this design: namely, participants would be highly practiced during the 20:80 condition on Day 2, and less so during the 50:50 condition on Day 1 . Thus, if we were to observe the predicted enhancement of the priming effect in the 20:80 condition on Day 2, one could argue that this was due to participants' performance simply improving over time as opposed to the redeployment of spatial attention. To rule out this possible explanation of our predicted results, before embarking on the experiment proper we ran an initial control experiment in which the target location probability was held constant at 50:50 across both testing days. If the properties of the masked congruence priming in the LVF are sensitive to the effects of practice, then we would expect to see differences in either the magnitude or onset of the MCE (or both) across days. To anticipate our results of this initial control experiment, the MCE did not vary across days in either hemifield, suggesting this index of face processing to be robust to the effects of practice.

\section{Experiment 1}

Our goal in Experiment 1 was to determine whether the masked congruence priming effect (particularly in the LVF) would improve as a function of exposure to the task. Participants attended two separate testing sessions within a 3-day period (i.e., the second session occurred either 1 or 2 days after the first). The task on each day was identical: classify the sex of the target face by reaching out to touch a response panel at the left or right edge of the screen. We used a fully crossed factorial design with the factors Visual Field (UVF vs. LVF) and Prime Type (congruent vs. incongruent). Importantly, this design was identical to the one planned for Experiment 2 (e.g., same number of trials, etc.), save that the predictability of the target's location did not vary across days. That is, the ratio of UVF to LVF targets was 50:50 on both Day 1 and Day 2. If the MCE is indeed sensitive to the effects of practice, then we should observe a stronger priming effect on Day 2 compared to Day 1.

\section{Experiment 1 methods}

\section{Participants}

A group of 14 individuals (seven males) gave informed consent to participate in Experiment 1 for financial compensation. All participants had normal or corrected-to-normal vision, and were identified as strong right-handers using the Edinburgh Handedness Inventory (Oldfield, 1971). The mean age was 26.5 years.

\section{Stimuli and apparatus}

Targets were five neutral faces of each sex drawn from the Psychological Image Collection at Stirling database (PICS, http://pics.psych.stir.ac.uk/), cropped to exclude the facial contour. No face contained obvious sex indicators (e.g., facial hair). Distractors were ten animal images presented within a similar cropped oval, one of which was selected at random on each trial. We used the SHINE toolbox written for Matlab to adjust the ten target and ten distractor items so that their mean luminance and contrast values were comparable (Willenbockel, Sadr, Fiset, Horne, Hosselin, \& Tanaka, 2010). We then used the same procedure to prepare an additional two faces (one male, one female) and two additional animal images to serve as masked primes and masked foils, respectively. These stimuli were considered "novel" in that they never appeared under unmasked conditions. The backward mask on each trial was randomly selected from a group of ten possible scrambled face images of the same mean luminance and contrast. All stimuli were $75 \times$ 100 pixels and subtended $3.44 \times 4.58^{\circ}$ of visual angle from a viewing distance of $65 \mathrm{~cm}$. Response panels marked " $\mathrm{M}$ " and 
"F" remained at the left and right edge of the screen throughout the trial; we counterbalanced the order of these positions across participants. We recorded each participant's reaching trajectories by fitting the their right hand with two light-emitting diode (LED) markers and sampling the position of these markers every 5 ms with an OptotrakCertus ${ }^{\circledR}$ motion capture system.

\section{Procedure and design}

The testing procedure was identical on both testing days. The participant sat in a dark room before a table with a touchscreen monitor fixed $50 \mathrm{~cm}$ from the front edge. Figure 1 presents the visual trial structure. Each trial frame consisted of two $75 \times$ 100 pixel panels, vertically displaced around a central fixation dot. We instructed participants to maintain their fixation on this central dot throughout the trial and reminded them of this between each block of trials. The trial commenced when the participant used their right index finger to depress a "start button" aligned with the body midline at the front of the table. The first frame contained identical chequerboard forward masks in each panel. After a variable duration, the prime face then appeared in either the upper or lower panel, accompanied by an animal foil in the opposite location. After $50 \mathrm{~ms}$, the target face replaced the prime face at the same location and was accompanied by a randomly selected animal distractor in the opposite panel. After $100 \mathrm{~ms}$, identical randomly selected backward masks onset in both panels and remained onscreen until the participant completed their classification by touching one of the two response panels located at the left and right edge of the screen. These conditions - namely the short prime duration and immediate masking by the target face - effectively mask the prime face so that its identity is typically not available for conscious report (Quek \& Finkbeiner, 2013, 2014a). For the manipulation of vertical hemifield presentation, on each trial the prime-target pair could appear in either the UVF or LVF with equal probability (50:50 UVF to LVF target location ratio). The prime was either the same sex or opposite sex as the target (i.e., congruent or incongruent), with the degree to which the prime's sex modulated the sex-categorization response to the target face serving as our index of nonconscious face perception. On Day 1, participants completed two practice blocks ( 80 trials total) followed by six experimental blocks ( 240 trials total). On Day 2, they completed one practice block (40 trials total) followed by 15 experimental blocks (600 trials total). All practice trials were discarded from later analyses.

On each trial, the participant had over $3 \mathrm{~s}$ to freely adjust their reaching trajectory and finalize their classification of the target by touching either the left or right response panel. However, although participants could complete the reaching movement in their own time, we imposed strict parameters regarding when they should initiate the movement. We required participants to begin reaching in response to an auditory go-signal on each trial - the third tone in a series of three ascending beeps (see Finkbeiner, Coltheart, \& Coltheart, 2014; Quek \& Finkbeiner, 2014a). The position of this gosignal varied in time with respect to target onset, such that on any trial the third beep could occur at one of five randomly selected durations following target onset $(0 \mathrm{~ms}, 75 \mathrm{~ms}$, $150 \mathrm{~ms}, 225 \mathrm{~ms}$, or $300 \mathrm{~ms}$ ). We defined Movement Initiation Time (MIT Latency) as the time in milliseconds from target onset until the finger's velocity reached $10 \mathrm{~cm} / \mathrm{s}$. We allowed MIT latencies up to $100 \mathrm{~ms}$ before and $200 \mathrm{~ms}$ after the beep signal. If the participant began their reaching movement outside this critical window, they received negative auditory (a loud buzz) and visual feedback (e.g., "Too Early!"), and the trial was aborted and cached for re-presentation at the end of the block. Trials with response-window failures during the representation stage were removed from all subsequent analyses (11.37 \% of all trials).

As in our previous study, we assessed the visibility of the masked prime items using a two-alternative forced-choice (2AFC) prime-detection task (conducted at the conclusion of the Day 2 testing session). This prime-visibility test included 160 trials which were identical to those used in the experiment proper, save that after reaching out to classify the target face, participants saw two faces (the real prime and a lure) and had to indicate which of them had been the prime on that trial. This prime-identification response was untimed, and position of the real prime and lure was counterbalanced across trials. Importantly, we instructed participants to maintain the same strategy for these prime-identification trials as they used in the experiment proper.

\section{Analysis methods}

Data preparation To prepare the reaching trajectories for analysis, we selected the $450 x y z$ samples between the points corresponding to $100 \mathrm{~ms}$ before movement onset, and $2150 \mathrm{~ms}$ after movement onset. ${ }^{1}$ At each sample within this epoch, we calculated $x$-velocity - a signed value which reflects the finger's velocity along the $x$-axis. Because participants indicate their sex-categorization decision along this leftright dimension (e.g., left for male; right for female), $x$-velocity at any given sample represents the finger's velocity in the correct direction. Positive $x$-velocity values indicate the finger

\footnotetext{
${ }^{1}$ We included the $100 \mathrm{~ms}$ leading up to movement onset to ensure that we considered the very start of the reaching movement on each trial. For instances in which the participant's reaching movement concluded sooner than $2,150 \mathrm{~ms}$ after movement onset, we repeated the $x y z$ coordinates from the final sample to make up the full number of samples for that trial.
} 


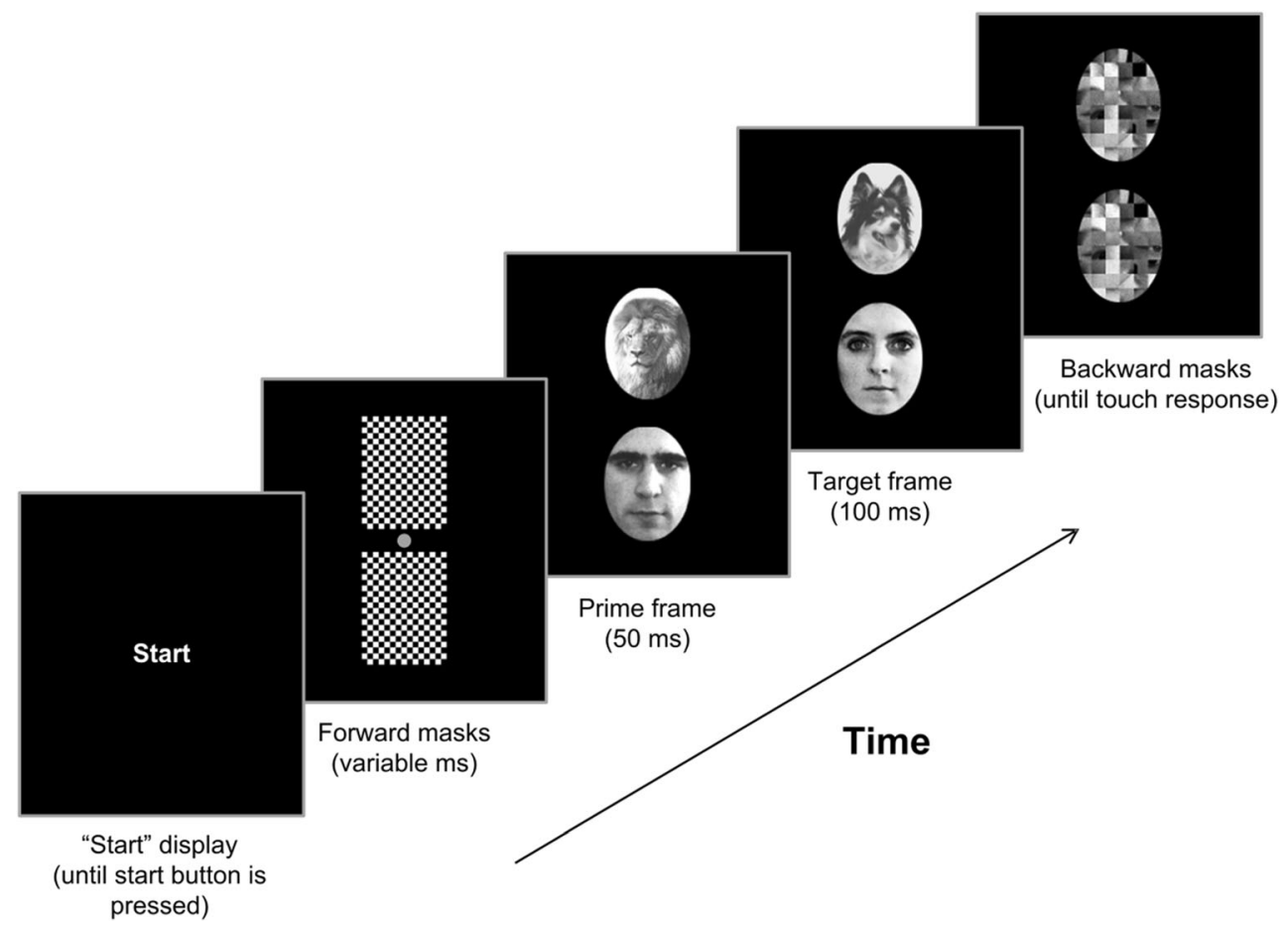

Fig. 1 Visual trial structure for Experiments 1 and 2. Each trial frame consisted of two $75 \times 100$ pixel vertically displaced panels. The trial began when the participant depressed a start button aligned with the body midline at the front edge of the testing table. Participants saw an initial fixation frame containing two identical chequerboard masks and a central dot they were required to fixate on. After a variable duration, the prime face appeared in either the top or bottom panel for $50 \mathrm{~ms}$, accompanied by an opposite animal foil. The subsequent frame contained the to-be-categorized target face in the same location as where the prime had appeared, with another animal distractor in the opposite panel. After $100 \mathrm{~ms}$, backward masks onset in both panels and remained onscreen until the participant completed their classification response is moving towards the correct response panel, and negative $x$ velocity values indicate that the finger is heading away from the correct response panel (i.e., in the incorrect direction). We smoothed the $x$-velocity profiles using a modified version of the Orthogonal Polynomial Trend Analysis (OPTA) procedure (Karayanidis, Provost, Brown, Paton, \& Heathcote, 2011; Woestenburg, Verbaten, \& Slangen, 1983), the details of which have been extensively reported elsewhere (Finkbeiner, Coltheart, \& Coltheart, 2014; Quek \& Finkbeiner, 2013, 2014a). As a final step, we averaged across the initial $300 \mathrm{~ms}$ of the reaching movement to produce a single representative value for each trial termed initial $x$-velocity. We limited our dependent measure to the initial segment of the trajectory because this initial portion best reveals the motor plan that participants had formulated just prior to movement initiation. This is important since we are interested in comparing the way in which participants' responses develop with stimulus-viewing time across different experimental manipulations. In the present case, the masked congruence effect (MCE) is reflected in higher initial $x$-velocities on congruent trials than incongruent trials. This pattern is analogous to the MCE reflected in response-time (RT) data (e.g., Finkbeiner \& Palermo, 2009; Naccache, Blandin, \& Dehaene, 2002), in that just as participants in button-press priming studies tend to press the correct button faster on congruent trials than incongruent, here participants move in the correct direction faster when the prime and target are associated with the same classification response.

Statistical analyses We analyzed all data using custom software written in R (www.r-project.org), implementing linear mixed-effects modelling (LMM; cf. Baayen, Davidson, \& Bates, 2008; Bates, 2005) using the lmer4 package (http:// lme4.r-forge.r-project.org, Bates, Maechler, \& Bolker, 2011). We evaluated the reliability of each effect of interest using an incremental model comparison procedure in which we used goodness-of-fit statistics (AIC, BIC, and Log Likelihood values; see Akaike, 1974; Schwarz, 1978) to determine which of two models fit our data better - a model which included the term under inspection, or the same model without this term. For each comparison, we selected the model which (a) minimized AIC and BIC, and (b) maximized the Log Likelihood. Below we report the results of this Likelihood ratio test and, where appropriate, also report the coefficients, standard errors (SE), and $t$-values for terms included in the final model selected. Our criterion for significance for individual fixed effects was an absolute $t$ ratio of 2.0, as per Kliegl, Masson, and Richter (2010). Although the degrees of freedom are not 
known exactly in LMM, the very large number of observations in the datasets used here and elsewhere mean that the $t$ distribution converges to the normal distribution. Thus, the criterion cutoff of two SEs corresponds well to the .05 significance criterion (see Finkbeiner et al., 2014; Kliegl et al, 2010; Masson \& Kliegl, 2013; Quek \& Finkbeiner, 2013, 2014a).

\section{Experiment 1 results}

\section{Accuracy}

The mean classification accuracy for Experiment 1 was $85.5 \%$. LMM analysis confirmed that a model including random slopes between Participant and Prime Type fit the binomial accuracy data better than a model including only random intercepts for Participant, $\chi^{2}(2)=155.82, p<.001$. We then verified that including the fixed effects of Prime Type, $\chi^{2}(1)$ $=18.27, p<.001$, Visual Field, $\chi^{2}(1)=72.74, p<.001$, and Day, $\chi^{2}(1)=28.80, p<.001$ also improved the model's fit. As can be seen in Fig. 2a, there was a strong MCE reflected in classification accuracy, in that participants were more likely to classify the target's sex correctly on congruent trials as compared to incongruent trials $(b=-0.74, S E=0.12, z=$ $-6.06, p<.001)$. Target classification was also more likely to be accurate for UVF trials than for LVF trials $(b=-0.43, S E$ $=0.05, z=-8.48, p<.001$ ) (see Fig. $2 b$ ), and on Day 2 compared to Day $1(b=0.31, S E=0.06, z=5.45, p<.001)$ (see Fig. 2c). Importantly, none of the two- or three-way interactions between these three experimental factors improved the model's fit ( $p>.05$ in all model comparison cases), indicating the best model of the accuracy data to be an additive one.

\section{Initial x-velocity}

The analysis procedure described above yielded 11,940 initial $x$-velocity values from 14 participants. We used LMM to firstly determine that a model with random slopes between Prime Type and Participant fit the initial $x$-velocity data better than one with only random intercepts for Participant, $\chi^{2}(2)=$ $1544.99, p<.001$. Including the fixed effect of Prime Type also significantly improved the model's fit, $\chi^{2}(1)=10.13, p<$ .005 . Just as with the accuracy data, there was a strong MCE evident in initial $x$-velocity, which was higher on average for congruent trials than incongruent trials $(b=-25.09, S E=6.57$ $t=-3.82)$. While the fixed effect of Visual Field did not significantly improve the fit of the model, $\chi^{2}(1)=0.40, p=$ .527 , we nonetheless retained this term in the model so as to inspect its interaction with other factors. This effect was reliable in the context of the final fitted model; however, with initial $x$-velocity observed to be higher on UVF trials than on LVF trials $(b=-8.41, S E=1.87, t=-4.50)$. Including the fixed effect of Day improved the model, $\chi^{2}(1)=91.53, p<$
.001 , with the final estimates indicating that initial $x$-velocity was higher on Day 1 than on Day $2(b=-12.13, S E=1.63 t=$ -7.46). Importantly for our purposes here, the interaction between Prime Type and Day did not improve the fit of the model, $\chi^{2}(1)=0.27, p=.603$, suggesting that the MCE evident in the initial stages of participants' reaching responses did not change as a function of exposure to the task. In contrast, there was a significant interaction between Prime Type and Visual Field, $\chi^{2}(1)=9.60, p<.01$. We followed this up by conducting a paired $t$-test between the congruent and incongruent conditions in each Visual Field ( $p$ values corrected using the False Discovery Rate, FDR). As can be seen in Fig. 3, there was a significant MCE in both the upper hemifield $(t(13)=3.80, p<.005$, Pearson's $r=.73)$ and the lower hemifield $(t(13)=3.10, p<.01$, Pearson's $r=.65)$. However, as the MCE above fixation was larger than the MCE below fixation $(25.97 \mathrm{~mm} / \mathrm{s}$ vs. $20.65 \mathrm{~mm} / \mathrm{s})$.

We also observed a significant Visual Field by Day interaction, $\chi^{2}(1)=13.40, p<.001$. On Day 1 , initial $x$-velocity was numerically higher for the UVF than the LVF. The reverse pattern was true on Day 2, however, follow-up paired $t$-tests (FDR (false-discovery rate corrected) of the Visual Field effect on each Day indicated that neither difference was statistically reliable ( $t<1$ in both cases). Our final model comparison verified that including the 3-way interaction between Prime Type, Visual Field, and Day did not improve the model's fit of the initial $x$-velocity data, $\chi^{2}(1)=2.20, p=.138$ (see Fig. 4). As can clearly be seen in Fig. 4C, the magnitude of MCE did not change from Day 1 to Day 2 for either the UVF or LVF. This suggests that participants' ability to extract sexinformation from the masked prime faces did not improve as a function of exposure to the task (i.e., practice).

\section{Prime detection}

To assess prime visibility, we used the $2 \mathrm{AFC}$ prime detection data to calculate a hit rate, false alarm rate, and $d$ ' value for each participant. The mean $d^{\prime}$ value was 0.07 . A one-sample $t$ test confirmed that this was not significantly different from zero $(t(13)=0.86, p=.404$, Pearson's $r=.23)$, suggesting participants in Experiment 1 had minimal awareness of the masked prime faces.

\section{Experiment 1 discussion}

We observed a strong MCE in Experiment 1, in that participant's classification reaching movements were both more accurate and more efficient for targets preceded by congruent primes compared to incongruent primes (i.e., same rather than opposite sex). We also found clear evidence of a UVF advantage for face-sex classification, in that participants were more accurate in categorizing the sex of UVF targets compared to LVF targets. Moreover, although both UVF and LVF prime- 
A

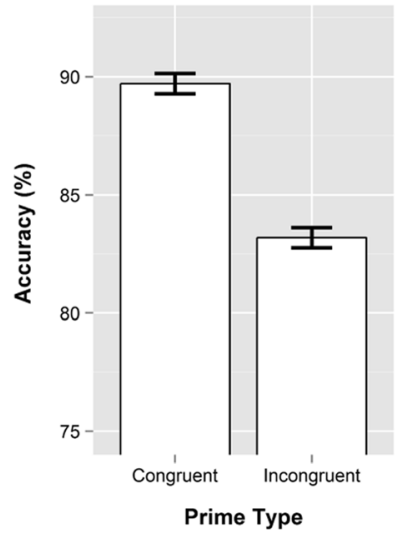

B

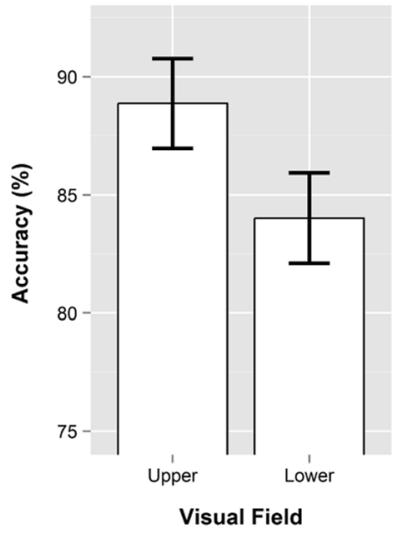

C

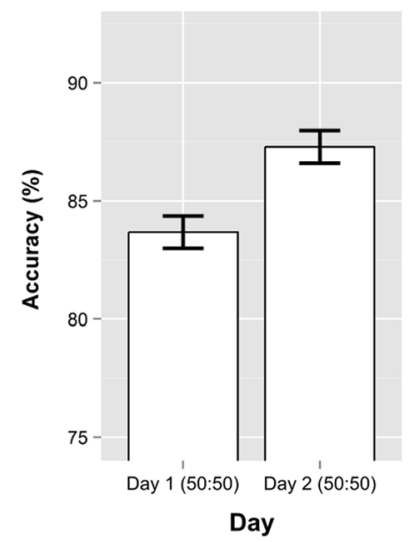

Fig. 2 Experiment 1 accuracy rates as a function of (a) Prime Type, (b) Visual Field, and (c) Day. Target classification was significantly more likely to be accurate when primes were congruent compared to incongruent; when the prime-target pair appeared in the upper visual field compared to the lower visual field; and on Day 2 compared to
Day 1. No interactions between these factors were reliable. Most importantly for our purposes here, our index of nonconscious face processing - the masked congruence effect - did not vary significantly across the testing days. Error bars are within-subjects standard error

process the sex information carried by the masked prime more efficiently when the prime appeared in the upper hemifield compared to the lower hemifield. Taken together, these results provide strong evidence for the emerging position in the literature that face processing, and in particular face-sex categorization, is superior in the upper hemifield relative to the lower hemifield (Liu \& Ioannides, 2010; Quek \& Finkbeiner, 2014a). In addition, and importantly for Experiment 2, we also found that the priming effect reflected in both participants' accuracy and initial reaching movements did not differ significantly between testing days for either hemifield. This suggests that participants' ability to extract the task-relevant sex information from masked faces did not improve as a function of exposure to the task (i.e., from the first to the second testing day).

\section{Experiment 2}

Armed with the knowledge that the MCE in our paradigm was not sensitive to the effects of practice across days, we were free to pursue the principal goal of the present study - to determine how voluntarily directed spatial attention would modulate the effect of vertical hemifield on masked face priming. Experiment 2 used an identical design to Experiment 1, save that here we manipulated target location predictability across days. On Day 1, the ratio of UVF to LVF targets was 50:50, such that target location was unpredictable to participants (just as in Experiment 1). On Day 2, however, we increased the ratio of LVF to UVF targets so that the target appeared below fixation on $80 \%$ of trials. We reasoned that participants would be sensitive to this increased probability of LVF targets and, after a period of learning, would direct their attention to the lower hemifield as a consequence. We 
Day 1 (50:50)
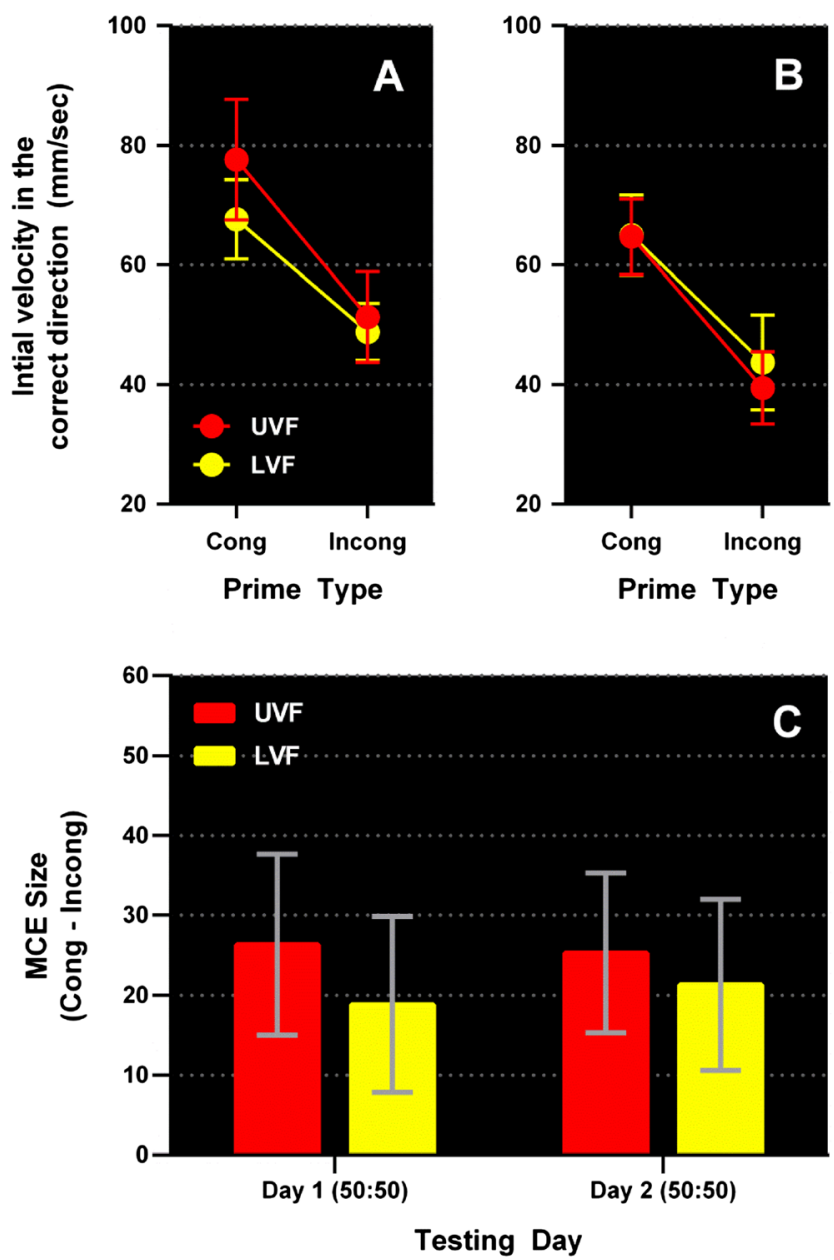

Fig. 4 (A and B) Experiment 1 initial $\mathrm{x}$-velocities shown as a function of Prime Type, Visual Field, and Day. The ratio of upper:lower visual field (UVF:LVF) targets were 50:50 on both days. The three-way interaction here was not significant, suggesting the priming effect in each Visual Field was similar across days. (C) The size of the masked congruence effect (MCE; congruent-incongruent) for the UVF and LVF on each testing day. Note that all $95 \%$ within-subjects confidence intervals (WSCIs) exclude zero, indicating there was a reliable MCE in all conditions. The magnitude of the MCE in each visual field was constant from Day 1 to Day 2. Error bars are $95 \%$ WSCIs, thus interbar overlap should not be interpreted by eye (see Cummings \& Finch, 2005)

predicted that covertly attending to the LVF would facilitate the priming effect in this hemifield, resulting in a stronger MCE for the LVF on Day 2 than on Day 1. Because we had already confirmed that the MCE in the LVF did not improve with task exposure in Experiment 1, we felt we would be able to attribute any improvement in the MCE in the LVF across testing days to our manipulation of target location probability, rather than simple practice effects. Of critical interest was what impact this manipulation would have on participants' ability to process masked prime faces above fixation. If the UVF advantage we and others have observed for face processing really is underpinned by an attentional bias towards the upper hemifield, then a redeployment of spatial attention to the LVF should yield an enhanced MCE in the LVF and a diminished MCE in the UVF.

\section{Experiment 2 methods}

\section{Participants}

We recruited a different group of 16 Macquarie University undergraduate students (seven males) to participate in Experiment 2 for course credit. All participants had normal or corrected-to-normal vision, and were identified as strong right-handers using the Edinburgh Handedness Inventory (Oldfield, 1971). The mean age was 18.81 years.

\section{Procedure and design}

We used the same stimuli, apparatus, trial structure, and testing procedure as described for Experiment 1. As before, we required participants to initiate their reaching movement in response to an auditory go-signal (the third beep in a series) which could occur either $0 \mathrm{~ms}, 75 \mathrm{~ms}, 150 \mathrm{~ms}, 225 \mathrm{~ms}$, or $300 \mathrm{~ms}$ after target onset. Participants completed 240 trials on Day 1 in which the prime and target faces appeared with equal probability (50\%) in either the upper or lower panel. On Day 2 , we included the same number of UVF trials as on Day 1 (i.e., 60 UVF-congruent, 60 UVF-incongruent), and included 480 LVF trials to yield a 20:80 ratio of UVF to LVF trials. There were 80 practice trials excluded from later analyses on Day 1 , and 40 on Day 2 . We assessed prime visibility at the end of the experiment proper on Day 2 using the same 2AFC task described for Experiment 1, in which we maintained the 20:80 UVF to LVF target ratio.

\section{Experiment 2 results}

\section{Accuracy}

Prior to analysis we removed all trials on which movement error occurred (11.81 \% of trials). Mean classification accuracy following this was $89.45 \%$. We then used LMM to verify that a model including random intercepts for each Participant, as well as random slopes between Participant and Prime Type, fit the binomial accuracy data better than a model with only random intercepts for each participant, $\chi^{2}(2)=414.38, p<$ .001. Including the fixed effect of Day significantly improved the model, $\chi^{2}(1)=40.96, p<.001$, in that there was a greater likelihood of participants classifying the target incorrectly on Day 2 than on Day $1(b=-0.35, S E=0.10, z=-3.47, p<$ $.001)$. The inclusion of Prime Type also improved the model, $\chi^{2}(1)=27.22, p<.001$, with a clear MCE reflected in participants' accuracy scores. Participants were significantly less 
likely to classify the target's sex correctly on incongruent trials $\left(M_{\mathrm{INCONG}}=86.95 \%\right)$ compared to congruent trials $\left(M_{\mathrm{CONG}}\right.$ $=96.22 \%)(b=-1.58, S E=0.19, z=-8.32, p<.001)$. As in Experiment 1, the interaction between Prime Type and Day did not improve the model, $\chi^{2}(1)=0.07, p=.786$, indicating that the effect of Prime Type did not vary from Day 1 to Day 2. Visual Field improved the model, $\chi^{2}(1)=$ $107.01, p<.001$; however, unlike in Experiment 1, this effect was qualified by testing Day, $\chi^{2}(1)=59.78, p<.001$. To follow up this two-way interaction we ran FDRcorrected paired $t$-tests between the UVF and LVF accuracy rates, separately for Day 1 and Day 2 . As can be seen in Fig. 5, when target location was unpredictable (i.e., Day 1), participants' classification accuracy was comparable between the upper $\left(M_{\mathrm{UPPER}}=88.84 \%\right)$ and lower visual fields $\left(M_{\text {LOWER }}=89.43 \%\right), t(15)=-0.24, p=.814$, Pearson's $r=.06$. In contrast, when participants could reliably expect the target to appear in the LVF (i.e., Day 2), classification accuracy was significantly higher in this region compared to the upper hemifield $\left(M_{\text {LOWER }}=94.38 \%\right.$ vs. $\left.M_{\mathrm{UPPER}}=85.24 \%\right), t(15)=-5.16, p<.001$, Pearson's $r$ $=.80$. To verify that the increased accuracy in the LVF on Day 2 did not come at a cost to target classification in the UVF, we then broke the interaction down the opposite way. FDR-corrected paired $t$-tests of the effect of Day for each Visual Field indicated that accuracy in the UVF was comparable between Day 1 and Day 2, $t(15)=1.67, p=.155$, Pearson's $r=.40\left(M_{\text {Day } 1}=88.84 \%\right.$ vs. $\left.M_{\text {Day } 2}=85.24 \%\right)$. In contrast, there was a significant improvement in accuracy for the LVF from Day 1 to Day 2, $t(15)=-2.68, p=$ .017 , Pearson's $r=.57\left(M_{\text {Day } 1}=89.43 \%\right.$ vs. $M_{\text {Day } 2}=$ $94.38 \%)$.

\section{Initial $x$-velocity}

As in Experiment 1, we calculated initial $x$-velocity by averaging the first $300 \mathrm{~ms}$ of each smoothed $x$-velocity profile. Below we subject these initial $\mathrm{x}$-velocities to statistical analysis, both (a) averaged across target viewing time and then (b) as a function of target-viewing time.

Analyses averaging across target-viewing time To model initial $\mathrm{x}$-velocity collapsing across target viewing times, we firstly verified that a model including both random intercepts for Participant and random slopes between Participant and Prime Type fit the data better than a model with only random intercepts $\chi^{2}(2)=2354.22, p<.001$. There was a significant fixed effect of Prime Type, $\chi^{2}(1)=13.97, p<.001$, in that initial $x$-velocity was higher for congruent trials compared to incongruent trials $(b=-27.82, S E=7.33, t=-3.80)$. The fixed effects of Visual Field, $\chi^{2}(1)=83.00, p<.001$, and Day, $\chi^{2}(1)=243.48, p<.001$, also improved the model's fit, with initial $x$-velocity being higher on average for UVF trials

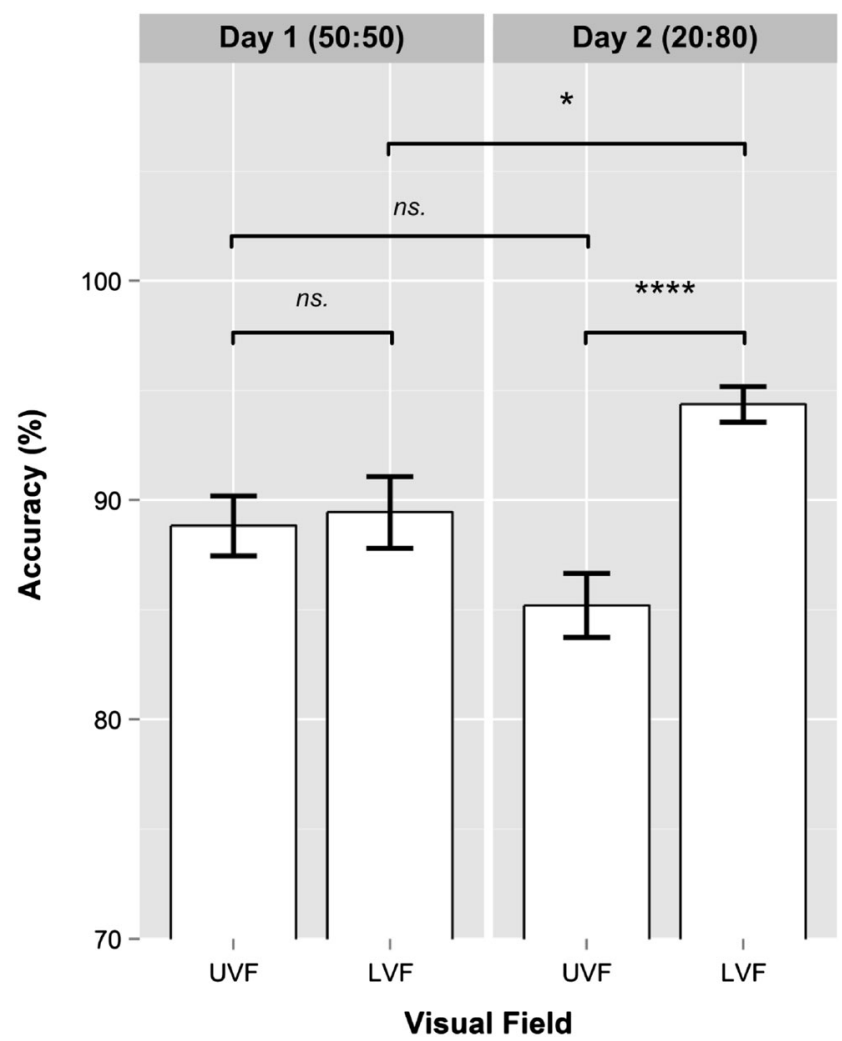

Fig. 5 Mean accuracy rates for Experiment 2 as a function of Visual Field and Day. When the ratio of upper (UVF) to lower (LVF) visual field targets was 50:50 (Day 1), accuracy rates were comparable between the upper and lower hemifields. In contrast, when the UVF to LVF location ratio was 20:80 (Day 2), accuracy was significantly higher in the LVF than in the UVF. This suggests our target location predictability manipulation was successful in encouraging participants to voluntarily direct spatial attention to the LVF on Day 2. Error bars are within-subjects standard error. ${ }^{*} p<.05 ; * * p .01 ; * * * p<.005$; $* * * * p<.001$ (two-tailed paired $t$ - tests, FDR (false-discovery rate) corrected)

compared to LVF trials $(b=-3.05, S E=2.08, t=-1.46)$, and on Day 2 compared to Day $1(b=14.67, S E=2.10, t=7.00)$. Regarding two-way interactions, including the Prime Type $\times$ Visual Field interaction did not improve the model's fit, $\chi^{2}(1)$ $=1.82, p=.177$; however, both the Prime Type $\times$ Day and Visual Field $\times$ Day interactions did, $\chi^{2}(1)=42.29, p<.001$ and $\chi^{2}(1)=5.81, p<.05$, respectively. Critically, and in contrast to Experiment 1, including the three-way interaction between Prime Type, Visual Field, and Day also significantly improved the model of Experiment 2 initial $x$-velocities.

We followed up the nature of this significant three-way interaction (presented in Fig. 6) by modelling the data separately for the upper and lower hemifields. The model for the UVF was characterized by a significant fixed effect of Prime Type, $\chi^{2}(1)=7.51, p<.01$, in which initial $x$-velocity was higher on congruent compared to incongruent trials $(b=$ $-27.67, S E=10.51, t=-2.63)$. There was also a significant fixed effect of Day, $\chi^{2}(1)=69.01, p<.001$, in that initial $x$ velocity for the UVF was higher on Day 2 than on Day $1(b=$ 
Day 1 (50:50)
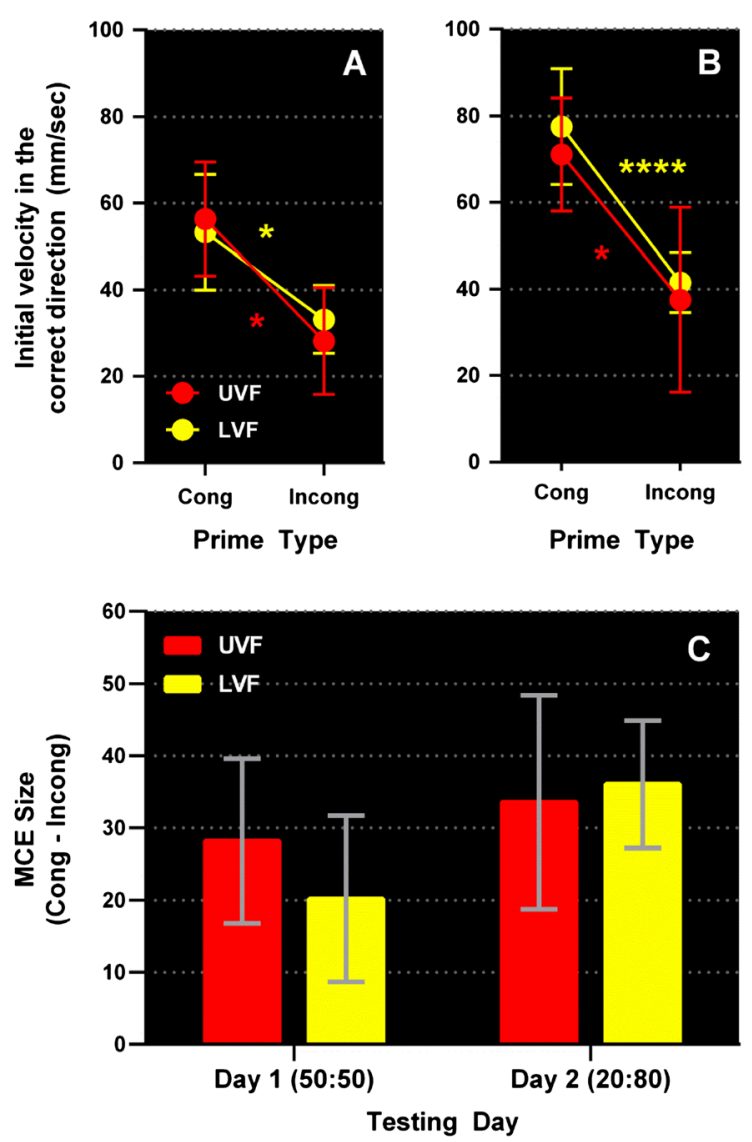

Fig. 6 (A and B) The significant three-way interaction in initial $\mathrm{x}$ velocity between Prime Type, Visual Field, and Day $\left({ }^{*} p<.05 ;{ }^{* *} p<\right.$ $.01 ; * * * p<.005 ; * * * * p<.001, \mathrm{FDR}$ (false-discovery rate) corrected), plotted also as (C) the masked congruence effect (MCE) difference score (congruent-incongruent) for the upper (UVF) and lower (LVF) visual field on each testing Day. As in Experiment 1, there was a reliable effect of prime type in all conditions. However, in the LVF (yellow bars), the MCE was clearly stronger on Day 2 than on Day 1, suggesting that prime-processing in the LVF was improved by participants attending to this region of space. Critically, the magnitude of the MCE in the UVF did not decrease from Day 1 to Day 2, indicating that attending away from this region did not impair prime-processing in the UVF in any way. Error bars are $95 \%$ within-subjects confidence intervals; inter-bar overlap should not be interpreted by eye (see Cummings \& Finch, 2005)

$15.58, S E=1.96, t=7.81)$. Importantly, including the interaction between Prime Type and Day also significantly improved our model of the initial $x$-velocity data for the UVF, $\chi^{2}(1)=5.83, p<.05$. As can be seen in Fig. 6C (red bars), although the MCE for the UVF was reliable on both Day 1 and Day 2 (as indicated by FDR-corrected paired $t$-tests: $t(15)$ $=2.85, p<.05$, Pearson's $r=.59$ and $t(15)=2.56, p<.05$, Pearson's $r=.55$ respectively), this effect was larger on Day 2 $(34 \mathrm{~mm} / \mathrm{s})$ than on Day $1(28 \mathrm{~mm} / \mathrm{s})$. The model for LVF initial $x$-velocities was similarly characterized by significant main effects of Prime Type, $\chi^{2}(1) 15.70, p<.001$, and Day, $\chi^{2}(1)$ $=198.83, p<.001$. Initial $x$-velocity was higher on congruent trials compared to incongruent trials $(b=-19.64, S E=6.86, t$ $=-2.87)$ and on Day 2 compared to Day $1(b=24.45, S E=$ $1.61, t=15.17)$. The interaction between these factors also improved the model, $\chi^{2}(1) 48.83, p<.001$. As can be seen in Fig. $6 \mathrm{C}$ (yellow bars), while the MCE in the LVF was reliable on both Day 1 and Day 2 (indicated by FDR corrected paired $t$-tests: $t(15)=3.21, p<.05$, Pearson's $r=.64$ and $t(15)=4.97$, $p<.001$, Pearson's $r=.79$ respectively), the magnitude of the effect was much larger on Day $2(36 \mathrm{~mm} / \mathrm{s})$ than on Day 1 $(20 \mathrm{~mm} / \mathrm{s})$.

For interest's sake, we also examined another aspect of the three-way interaction between Prime Type, Visual Field, and Day by modelling the data separately for Day 1 (50:50) and Day 2 (20:80). To summarize these analyses briefly, when target location was unpredictable (i.e., Day 1), there was a significant interaction between Prime Type and Visual Field which indicated the MCE to be larger in the UVF than the LVF $(28 \mathrm{~mm} / \mathrm{s}$ vs. $20 \mathrm{~mm} / \mathrm{s}), \chi^{2}(1)=7.91, p<.005$. In contrast, on Day 2 this interaction was not reliable, $\chi^{2}(1)=1.52$, $p=.218$, indicating that the magnitude of the MCE did not differ significantly between the hemifields on Day 2 when participants could expect the target to appear more frequently in the LVF $(34 \mathrm{~mm} / \mathrm{s}$ in the UVF vs. $36 \mathrm{~mm} / \mathrm{s}$ in the LVF).

Analyses taking target-viewing time into account In addition to comparing the magnitude of the MCE across experimental conditions, in Experiment 2 we wanted to examine whether the timecourse of this effect varied as a function of vertical hemifield or target location probability. To do so, we took advantage of the fact that our auditory go-signal manipulation ensured that participants' reaching trajectories would be initiated across a very wide range of MIT latencies (from $-100 \mathrm{~ms}$ before to $500 \mathrm{~ms}$ after target onset). Since MIT latency reflects the amount of time the participant has to process the critical stimuli prior to commencing their classification response, examining initial $x$-velocity as a function of MIT latency enables us to observe the MCE as it unfolds in stimulus-processing time (i.e., at less than $500 \mathrm{~ms}$ from target onset). The OPTA procedure we implemented to smooth our data enabled us to take account of this relationship between initial $x$-velocity and target-viewing time, depicted in Fig. 7. Here we have used MIT to group the $x$-velocity profiles into 20 bins of equal proportion (i.e., semi-deciles, see Fig. 7a) and calculated an average $x$-velocity profile for each of these MIT Quantiles (see Fig. 7b). The effect of target-viewing time on the unfolding of the trajectory response is plain in this figurethe longer participants wait to begin their reaching response, the faster their finger moves in the correct direction. That is, the more time participants have to process the stimuli before initiating their movement, the more efficient the initial stages of their classification response is.

To understand the timecourse of the MCE reflected in participants' reaching trajectories, we examined initial $x$-velocity 
Fig. 7 Examining $x$-velocity as a function of target-viewing time. (a) The distribution of movement initiation time (MIT) Latencies from target onset (i.e., a distribution of target-viewingtimes). We used Orthogonal Polynomial Trend Analysis (OPTA) to smooth the $x$-velocity profiles and grouped these into 20 bins (quantiles) ranked by MIT latency. (b) Mean $x$-velocity profiles by MIT Quantile. Darker colors indicate trials with short MIT Latencies (beginning at the first Quantile); lighter colors correspond to the longest MIT Latencies (20th Quantile). Note the clear effect of MIT Latency: the longer participants wait to begin moving, the faster the finger moves in the correct direction during the reaching response itself

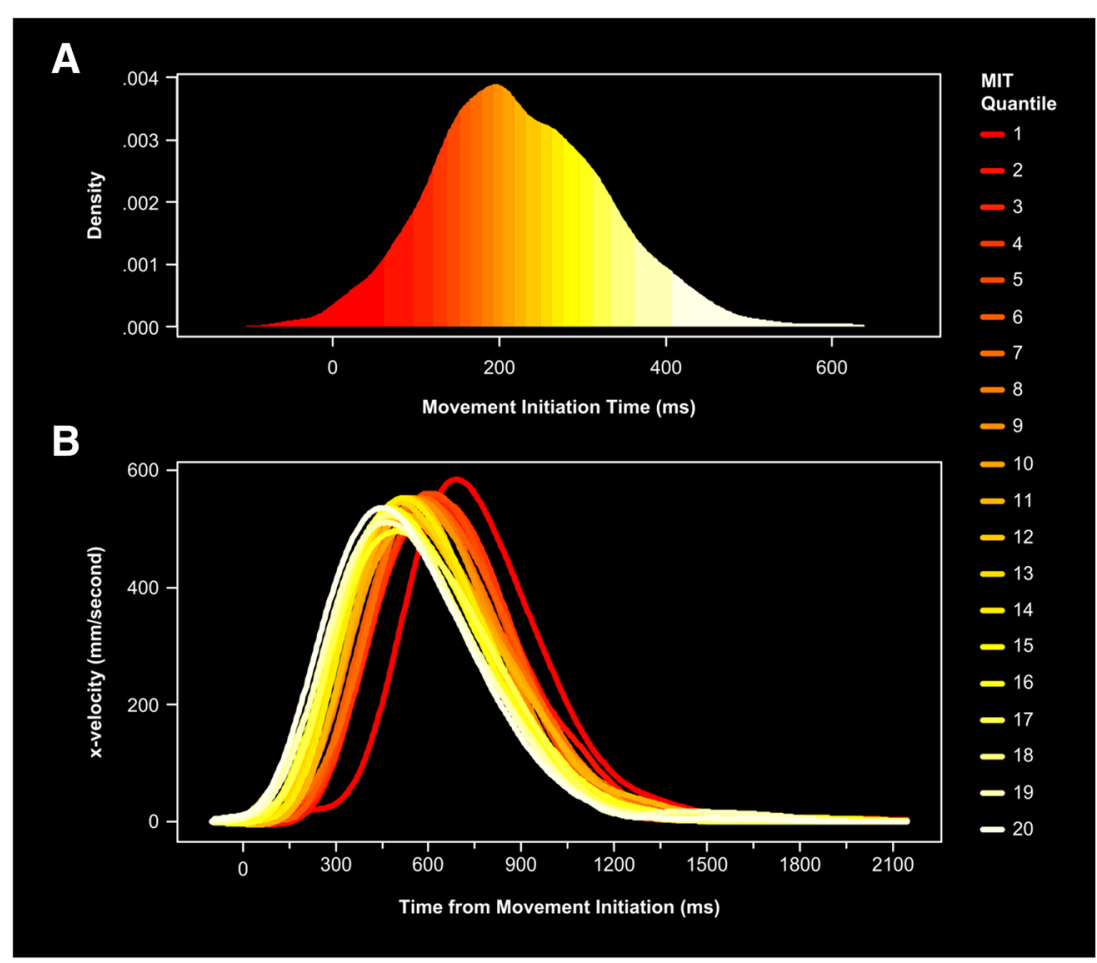

as a function of target-viewing time by including MIT Quantile as a factor in our LMM analyses. First, we verified that a model including random slopes between participant and Prime Type fit the initial $x$-velocity data better than a model with only random intercepts for each subject, $\chi^{2}(2)=$ $10737.41, p<.001$. Including the fixed effect of MIT Quantile further improved the model's fit, $\chi^{2}(1)=24.37, p<.001$. As is clear in Fig. 8, conditional mean initial $x$-velocity values increase dramatically as a function of target-viewing time (i.e., MIT latency, $b=4.75, S E=0.80, t=5.94)$. This indicates that the longer participants viewed the target prior to commencing their classification response, the faster their finger moved in the correct direction during the initial portion of the reaching movement. We then confirmed the presence of a strong MCE, evident in the significant fixed effect of Prime Type, $\chi^{2}(1)=$ $3815.40, p<.001$. Initial $x$-velocity was significantly higher on average for congruent trials compared to incongruent trials ( $b$ $=-20.55, S E=0.94, t=-21.84$ ) (see Fig. 8). Including the fixed effect of Day also improved the model, $\chi^{2}(1)=726.82, p$ $<.001$, as did the fixed effect of Visual Field, $\chi^{2}(1)=222.98, p$ $<$. 001. Initial $x$-velocity was reliably higher on UVF trials compared to LVF trials $(b=-2.51, S E=1.12, t=-2.24)$. To elucidate the timecourse over which these experimental effects emerged, we examined their interactions with MIT Quantile. We observed a significant Prime Type $\times$ MIT Quantile interaction, $\chi^{2}(1)=199.64, p<.001$, indicating that the magnitude of the MCE depended on target-viewing time. As may be seen in Fig. 8, the longer participants have to process the prime-target pair prior to initiating their classification response, the larger the MCE evident in the initial stages of the reaching movement. In other words, the ability of the prime to influence the initial stages of the classification response grew as a function of target-viewing time.

Next, we verified that the effect of target-viewing time on priming was further qualified by vertical hemifield (i.e., significant Prime Type $\times$ MIT Quantile $\times$ Visual Field interaction, $\left.\chi^{2}(2)=133.09, p<.001\right)$. Lastly, we determined that the modulation of the MCE's timecourse by vertical hemifield depended on the ratio of UVF to LVF targets (i.e., significant Prime Type $\times$ MIT Quantile $\times$ Visual Field $\times$ Location Predictability interaction, $\left.\chi^{2}(4)=400.96, p<.001\right)$. We followed up the nature of this four-way interaction (presented in Fig. 9) by modelling initial $x$-velocity separately for each testing day.

LMM analysis for Day 1 indicated significant fixed effects of MIT Quantile and Prime Type ( $p<.001$ in both cases), but not Visual Field, $\chi^{2}(1)=0.58, p=.448$. We nevertheless retained this factor in the model so as to inspect its interaction with other factors. Next, we verified the inclusion of the twoway interaction between Prime Type and MIT Quantile and determined that this was further qualified by Visual Field ( $p<$ .001 in both cases). Because we obtained the critical Prime Type $\times$ Quantile $\times$ Visual Field interaction, we were able to conduct a paired $t$-test between the congruent and incongruent conditions at each MIT Quantile, separately for the upper and lower hemifields. In the UVF (see Fig. 9A), the FDRcorrected $p$-values were significant from the 6 th to the 20th MIT Quantile - indicating that a participant's initial reaching movements reflected a significant MCE from $165 \mathrm{~ms}$ of 


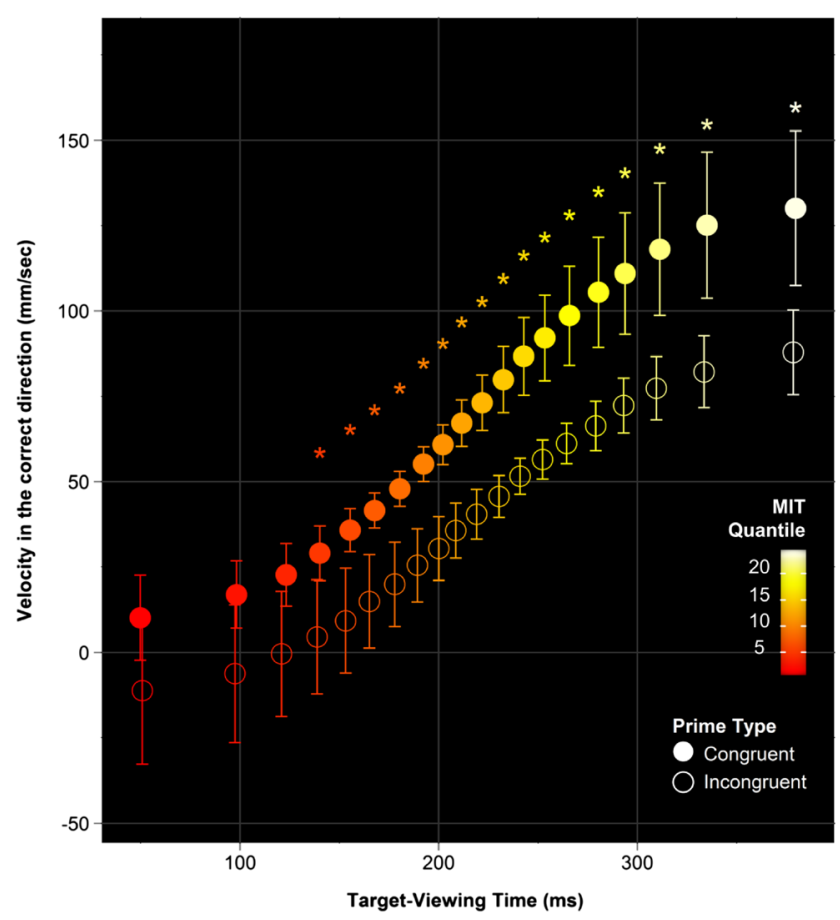

Fig. 8 Initial $x$-velocities as a function of movement initiation time (MIT) Quantile and Prime Type. Initial $x$-velocity reflects the finger's velocity in the correct direction averaged across the first $300 \mathrm{~ms}$ of the classification response. There was a strong effect of target-viewing time (i.e., MIT Quantile) in that the longer participants processed the primetarget pair before initiating their reaching response, the faster they moved in the correct direction during its initial stages. There was also a clear masked congruence effect (MCE), with congruent trials (filled circles) producing higher initial $x$-velocity values than incongruent trials (open circles). As is clear in the figure above, the prime's ability to influence the response to the target appeared to grow as a function of target-viewing time. Asterisks denote .05 significant contrasts between congruent and incongruent values at each MIT Quantile (FDR (false-discovery rate) corrected). Error bars are $95 \%$ within-subjects confidence intervals; inter-bar overlap should not be interpreted by eye (see Cummings \& Finch, 2005)

target-viewing time onwards. In contrast, primes presented in the LVF did not elicit a reliable MCE until the 8th MIT Quantile, at around $195 \mathrm{~ms}$ of target-viewing time (see Fig. 9C). Thus, on Day 1, when target location was unpredictable for participants, we observed a clear UVF advantage for masked face processing, with the MCE emerging some $30 \mathrm{~ms}$ earlier in stimulus-processing time for UVF face stimuli than for LVF face stimuli.

LMM analysis for Day 2 confirmed the same fixed effects and interactions as observed for Day $1(p<.001$ in all model comparison cases). Most importantly, the significant three-way interaction indicated that emergence of the MCE across MIT Quantile depended on Visual Field. As for Day 1, we further examined this interaction by conducting a paired $t$-test comparison of the congruent and incongruent conditions at each MIT Quantile, separately for each hemifield. As can be seen in Fig. 9B, the MCE in the UVF was reliable from the 6th to the 20th
MIT Quantile, or from approximately $165 \mathrm{~ms}$ of targetviewing time onwards. In contrast, the MCE elicited by prime-target pairs presented in the LVF was reliable at all MIT Quantiles, from around $53 \mathrm{~ms}$ of target-viewing time onwards (see Fig. 9D). Thus, on Day 2, in which participants could reliably anticipate the target's location and direct their attention appropriately in response, we observed an LVF advantage for masked face processing. Here primes presented in the LVF elicited a priming effect at an earlier stage of stimulus processing than primes presented in the UVF - the opposite pattern to that observed on Day 1, when target location was unpredictable. Of critical importance for present purposes is the comparison between Day 1 and Day 2 for the UVF. Looking across Figs. 9A and B, it is clear that face processing efficiency in the UVF did not suffer when participants covertly attended to the LVF. That is, the MCE in the UVF emerged at the same stage of stimulus-processing on both Day 1 and Day 2 (i.e., around 165 ms of target-processing time), suggesting that directing participants' covert attention away from the upper hemifield did not interfere with participants' ability to process masked faces in this region.

\section{Prime detection}

As in Experiment 1, to assess prime visibility we calculated $d^{\prime}$ from each participant's $2 \mathrm{AFC}$ prime detection data. Here the mean $d$ ' value was $0.11(S D=0.13)$, which a one-sample $t$-test determined was significantly greater than zero $(t(15)=3.37$, $p<.01$, Pearson's $r=.66$ ). This suggests that the participants in Experiment 2 may have had some partial awareness of the masked prime items, likely owing to our manipulation of target location predictability. That is, because participants voluntarily attended to the lower hemifield in response to the increased probability of targets in this region, masked primes presented in the LVF were likely to have been subject to focused spatial attention.

\section{Experiment 2 discussion}

Just as in Experiment 1, we observed a strong MCE in both participants' accuracy and initial reaching movements. We also replicated the UVF advantage for facesex categorization in that when participants could not reliably predict the target's location (i.e., on Day 1), the magnitude of the MCE reflected in the initial stages of their reaching movement was reliably larger for UVF prime-target pairs than for LVF pairs. Moreover, the analysis that took target-viewing time into account revealed that the MCE under unpredictable target location conditions emerged earlier during stimulus-processing time when the face stimuli appeared in the UVF compared to 


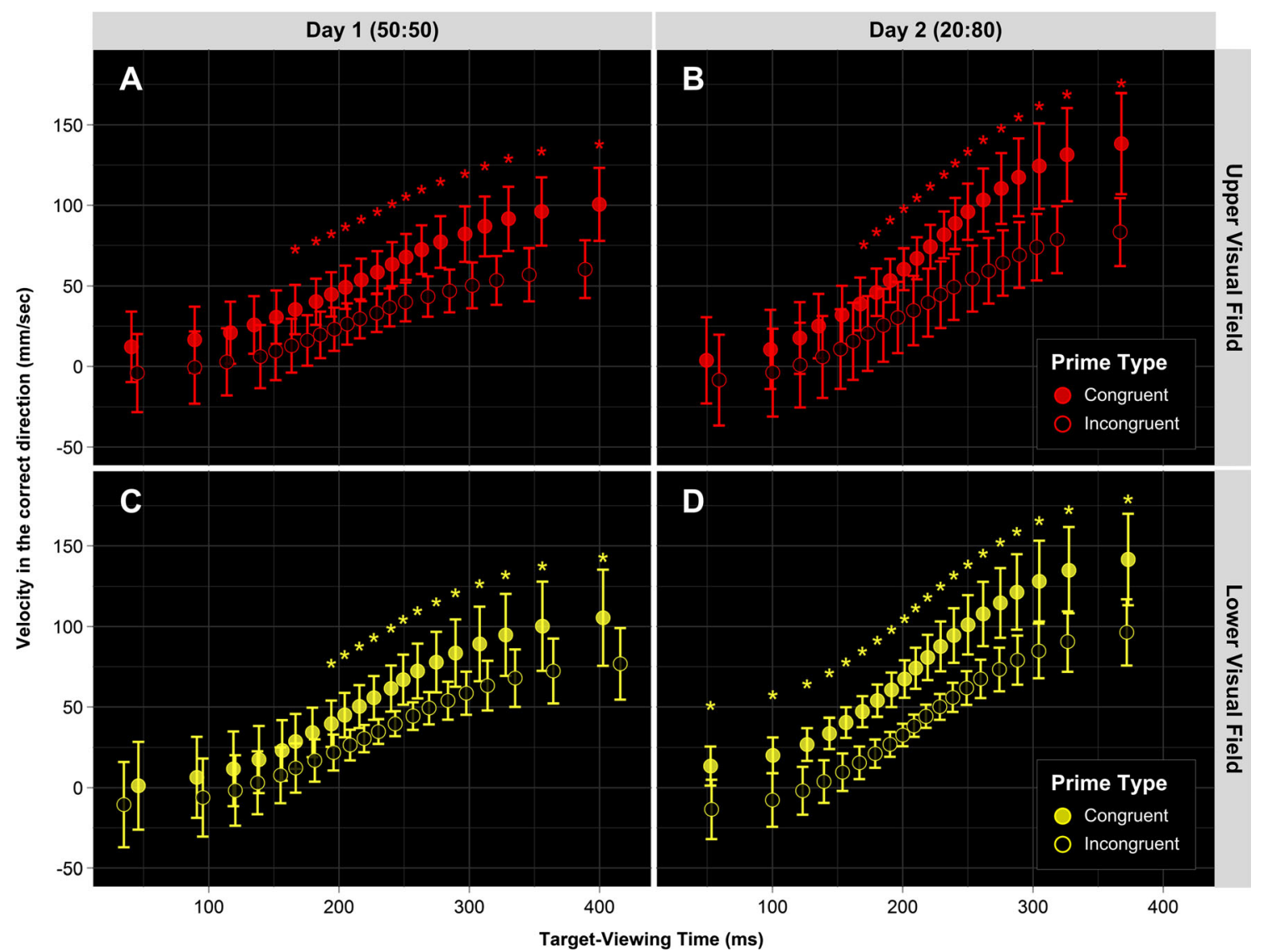

Fig. 9 Initial $x$-velocity as a function of Prime Type, movement initiation time (MIT) Quantile, Visual Field, and Day. (A) On Day 1 (50:50 target location ratio), the masked congruence effect (MCE) in the upper visual field (UVF) was significant from $\sim 165 \mathrm{~ms}$ of target-viewing time onwards, whereas $(\mathbf{C})$ lower visual field (LVF) primes did not elicit a reliable MCE until $\sim 195 \mathrm{~ms}$ of target-viewing time. The reverse was true on Day 2, in which participants could reliably expect the target to appear in the LVF more often than the UVF (80:20 ratio). Here the MCE

the LVF ( $\sim 165 \mathrm{~ms}$ vs. $\sim 195 \mathrm{~ms}$ of stimulus-processing time). That masked prime items influenced the target classification response at an earlier stage of stimulusprocessing when presented above - compared to below - fixation would suggest that face-sex categorization is more efficient in the UVF than the LVF.

The effect of vertical hemifield on masked-face processing was very different, however, when participants could reliably expect the target to appear in the lower half of the display and could direct their spatial attention appropriately in response (i.e., on Day 2). Here accuracy was better for LVF targets than for UVF targets. Moreover, the magnitude of the MCE in the LVF was significantly larger on Day 2 than on Day 1. This suggests that voluntarily directing their spatial attention towards the LVF improved participants' ability to process masked prime faces presented in this region. This interpretation is further supported by the finding that LVF primes influenced the initial stages of target classification at a much earlier point during was significant from $\sim 53 \mathrm{~ms}$ onwards in the LVF (D) and from $\sim 165 \mathrm{~ms}$ onwards in the UVF (B). A comparison of panels (B) and (D) suggests that directing spatial attention away from the UVF did not impair participants' ability to process masked faces presented in this region. Asterisks denote .05 significant contrasts between congruent and incongruent values at each MIT Quantile (FDR corrected). Error bars are $95 \%$ within-subjects confidence intervals; inter-bar overlap should not be interpreted by eye (see Cummings \& Finch, 2005)

stimulus-processing when participants voluntarily attended to the lower hemifield (i.e., on Day 2 compared to Day 1). By itself, this result is perhaps not altogether surprising indeed, there is good reason to expect that attending to the prime's location should facilitate processing and increase its ability to the response to the target (see Quek \& Finkbeiner, 2013). What is surprising, however, is that the increase in performance for LVF faces was not coupled with a commensurate decrease in performance for UVF faces. Target classification accuracy for UVF targets was comparable across testing days, and the MCE in the UVF was neither diminished nor delayed on Day 2 compared to Day 1 , emerging at the same time during stimulus processing on both testing days ( $\sim 165 \mathrm{~ms})$. Taken together, these results suggest that while directing covert spatial attention to the lower hemifield clearly confers a benefit on the processing of LVF faces, face processing in the UVF appears to be unaffected by participants' deployment of spatial attention to the opposite hemifield. 


\section{General discussion}

This paper establishes three important findings. First, we have demonstrated that under unpredictable target location conditions, face-sex categorization exhibits a clear UVF advantage. We observed this robust advantage in two separate groups of participants in both the magnitude and the timecourse of the MCE. Participants in Experiment 1 also showed this effect in sex-categorization accuracy. These results replicate an earlier study (Quek \& Finkbeiner, 2014a) and they are also highly consistent with findings from the neurophysiological literature showing that faces presented above fixation activate regions such as the left fusiform face area (FFA), left occipital face area (OFA), and medial prefrontal cortex (mPFC) earlier than faces presented below fixation (Liu \& Ioannides, 2010). Taken together, these findings strongly suggest that the visual system processes faces more efficiently in the upper hemifield than in the lower hemifield.

Second, we have shown that voluntarily directed spatial attention reliably modulates the ability of masked faces to influence the overt response to the target face. Masked primes in our study influenced the target classification response to a greater extent, and at an earlier stage of stimulus-processing, when participants could predict the likely location of the upcoming target and direct their attention to this location. This finding represents an important contribution to the masked priming literature, in that as far as we are aware, it is the first demonstration that endogenously-directed spatial attention modulates the masked priming effect for faces. The finding also echoes and complements our previous demonstrations that exogenously-oriented spatial attention facilitates the processing of masked face primes (Quek \& Finkbeiner, 2013, 2014a).

Third, and most importantly, the present paper dispels the possibility that the UVF advantage for face processing is underpinned by an upward bias in voluntarily-directed spatial attention. If this were the case, then masked face processing in the UVF would have been negatively affected when participants voluntarily attended to the opposite hemifield, as they did on Day 2 of our second experiment. Instead, we found that participants' ability to extract the task-relevant sex information from masked faces in the upper hemifield did not vary as a function of our manipulation of voluntarily directed spatial attention. This key finding strongly undermines the possibility that the UVF advantage for face-sex processing we report here and elsewhere (Quek \& Finkbeiner, 2014a) is driven by an upward bias in spatial attentional orienting (see Bradshaw et al., 1985; Drain \& Reuter-Lorenz, 1996; van Vugt et al., 2000). The implications of these findings for the masked priming, face perception, and broader object recognition literatures are discussed below.
The upper-hemifield advantage for face processing: Not a product of attentional bias

Although vertical asymmetry in face perception has received far less attention than the well-documented laterality effects for faces, there is nonetheless increasing evidence to suggest that face processing may be superior in the upper hemifield relative to the lower hemifield (Coolican, Eskes, McMullen, \& Lecky, 2008; Kessler \& Tipper, 2004; Liu \& Ioannides, 2010). In a recent study, we provided what might be considered the strongest evidence to date for a UVF advantage in face perception, demonstrating that masked faces affect the sex-categorization of a target face at an earlier stage of stimulus processing when presented in the UVF compared to the LVF (Quek \& Finkbeiner, 2014a). In the present study, we have replicated this finding in two separate groups of individuals, providing strong support for the existence of an upper hemifield advantage in face-sex processing. The discovery has significant implications for the face-perception literature, as where researchers have long tried to take account of laterality artefacts in their designs, thus far there appears to have been little consideration given to the impact of vertical anisotropy in face-perception tasks. The finding also qualifies the characterization of sex categorization as a highly robust instance of object recognition (Bruce \& Young, 1998; Wild et al., 2000). While this process is unquestionably efficient, we have highlighted that this aspect of face perception is nevertheless still subject to modulation by factors such as spatial location and spatial attention. A natural extension of this finding, and one that is already hinted at in the literature, would be to examine whether other aspects of face perception, such as identity or emotion recognition, exhibit a similar upper hemifield advantage (Felisberti \& McDermott, 2013; Kessler \& Tipper, 2004; Liu \& Ioannides, 2010). Given that some have suggested that visual field biases for face processing are distinct to individual participants (Afraz et al., 2010), another intriguing line of future enquiry is how individual differences in visual field shape (Fortenbaugh, Silver, \& Robertson, 2015) might influence the UVF advantage for faces we have documented here.

Importantly, although the mechanism underlying this vertical asymmetry for faces remains unclear, we have provided strong evidence here to discredit the possibility that the upper hemifield advantage for face processing is driven by an upward bias in voluntarily directed spatial attention. When participants' directed their spatial attention away from the upper hemifield, as they did when targets appeared in the lower visual field on $80 \%$ of the trials, their ability to process faces presented in the UVF was unaffected. It is worth noting, however, that voluntarily directed spatial attention is not the only top-down process capable of modulating basic perceptual processing. Where endogenously directed spatial attention prioritises stimulus processing on the basis of task demands, 
expectation can constrain visual interpretation on the basis of prior knowledge (Summerfield \& Egner, 2009) by sharpening sensory representations (Kok, Jehee, \& de Lange, 2012). It is well established, for example, that object detection is facilitated by a congruent context (e.g., a coffee cup in a kitchen scene will be found faster than the same cup in a beach scene) (Davenport, 2007; Fize, Cauchoix, \& Fabre-Thorpe, 2011). Moreover, Biederman has singled out both probability and spatial position as important relations which affect object recognition in scenes (Biederman, Mezzanotte, \& Rabinowitz, 1982). Thus, it is reasonable to think that our wealth of experience encountering faces in extrapersonal space could well have engendered in us a very strong expectation that face stimuli are more likely to appear above fixation than below. Just as expectation of object category is known to increase the efficiency with which category exemplars are processed (Puri \& Wojciulik, 2008), presumably a perceptual "prior" which also encompasses spatial location might be expected to facilitate exemplar processing in an anisotropic manner. Importantly, while attention and expectation are often intertwined, it could be the case that a perceptual prior for faces is overlearned and thus relatively less flexible. With this possibility, the probabilistic manipulation of target location used in our second experiment might have effectively guided participants' voluntarily directed spatial attention, while simultaneously having no effect on their pre-existing expectation. The possibility of a relatively inflexible prior expectation about face location could explain the UVF advantage observed when participants have no cause to preferentially direct their spatial attention to either hemifield (i.e., when target location is unpredictable). This possibility could also explain why face processing remains unaffected in the UVF in experimental contexts in which participants voluntarily direct their attention to the lower visual field. That is, whereas spatial attention can be flexibly controlled and directed to facilitate stimulus processing in predicted target regions, perhaps the well-learned perceptual prior regarding faces remains unchanged, allowing face processing in the expected region to proceed similarly with or without spatial attention. Intriguingly, there is already some indication that the strength of face and body representations in different parts of the visual field appears to be modulated by the frequency with which the stimuli occur in those locations (Chan, Kravitz, Tryuong, Arizpe, \& Baker, 2010).

Alternatively, it may be that a structural account is best equipped to explain why face processing exhibits a UVF advantage. As others have already alluded to (Kravitz et al., 2010; Kravitz, Saleem, Baker, Ungerleider, \& Miskin, 2013), since the representation of the upper hemifield in early visual cortex is predominantly contained below the calcarine sulcus (Sereno et al., 1995; Zeki, 1969), it may be that presenting faces in the UVF enables more efficient transfer of information to the ventral object recognition systems contained in the temporal lobe, such as the posterior fusiform
(Ishai, Ungerleider, Martin, Schouten, \& Haxby, 1999; Kravitz et al., 2010; Martin, Wiggs, Ungerleider, \& Haxby, 1996). Conversely, transfer to these ventral regions may take slightly longer from the dorsal regions of striate and extrastriate cortex, where information presented to the LVF is initially projected. We note, however, that at present this account of the UVF advantage in face perception remains speculative - it is clear that there are still important steps to be taken in furthering our understanding of vertical asymmetries in object-recognition. Perhaps the foremost question of interest is whether the UVF advantage is specific to face processing, or whether it may in fact extend to other forms of object recognition, as early reports suggest it could (see Quek \& Finkbeiner, 2014b).

\section{Endogenous attention modulates masked face processing}

Where the modulatory effects of voluntarily directed spatial attention on the neural response to face stimuli are well described in the neurophysiological literature (Holmes, Kiss, \& Eimer, 2006; Holmes, Vuilleumier, \& Eimer, 2003; Pessoa, McKenna, Gutierrez, \& Ungerleider, 2002; Vuilleumier, Armony, Driver, \& Dolan, 2001; Wojciulik, Kanwisher, \& Driver 1998), behavioral investigations to date have often failed to observe an impairment in task performance resulting from directing spatial attention away from a face's location. For example, Reddy and colleagues showed that neither face identification nor face-sex classification of peripheral masked faces suffer when spatial attention is engaged centrally by a demanding discrimination task (Reddy, Reddy, \& Koch, 2006; Reddy, Wilken, \& Koch, 2004). At first glance, it may appear that our findings do not support the conclusions of Reddy and colleagues. This is because we have shown that face perception does indeed benefit from endogenously directed spatial attention, just as it does from exogenously captured attention (Quek \& Finkbeiner, 2013). But we suggest, in fact, that our results are consistent with those of Reddy and colleagues. Just as participants in their studies were able to reliably identify both identity and sex even when attention was directed elsewhere, participants in our study remained perfectly able to extract the sex-information from masked face primes in the UVF when attention was directed the LVF.

\section{Conclusion}

There is increasing evidence to suggest that face perception is supported better at above-fixation locations than at belowfixation locations. The present study further establishes this finding by showing that face-sex categorization is more efficient in the UVF than the LVF. It has been suggested that the UVF advantage might be due to participants possessing a strong upward attentional bias in categorization tasks. The 
purpose of this study was to test this possibility. Our primary conclusion is that the UVF advantage is not due to an upward bias in voluntarily directed spatial attention. Specifically, masked faces presented in the upper hemifield engaged cognitive processes to the same degree even when spatial attention had been successfully deployed elsewhere.

Acknowledgments The authors are grateful to Irene Chork for her assistance in collecting behavioral data. This work was supported in part by an Australian Research Fellowship (FT120100830) to MF from the Australian Research Council.

\section{References}

Afraz, A., Pashkam, M. V., \& Cavanagh, P. (2010). Spatial heterogeneity in the perception of face and form attributes. Current Biology, 20(23), 2112-2116.

Akaike, H. (1974). A new look at the statistical model identification. IEEE Transactions on Automatic Control, 19(6), 716-723.

Amenedo, E., Pazo-Alvarez, P., \& Cadaveira, F. (2007). Vertical asymmetries in pre-attentive detection of changes in motion direction. International Journal of Psychophysiology, 64(2), 184-189.

Baayen, R. H., Davidson, D. J., \& Bates, D. M. (2008). Mixed-effects modeling with crossed random effects for subjects and items. Journal of Memory and Language, 59, 390-412.

Bates, D. (2005). Fitting linear mixed models in R. R News, 5, 27-30.

Bates, D., Maechler, M., \& Bolker, B. (2011). Linear mixed-effects models using S4 classes. $R$ package version 0.999375-42, http:// lme4.r-forge.r-project.org/.

Biederman, I., Mezzanotte, R. J., \& Rabinowitz, J. C. (1982). Scene perception: detecting and judging objects undergoing relational violations. Cognitive Psychology, 14(2), 143-177.

Bradshaw, J. L., Nettleton, N. C., Nathan, G., \& Wilson, L. E. (1985). Bisecting rods and lines: effects of horizontal and vertical posture on left-side underestimation by normal subjects. Neuropsychologia, 23, 421-425.

Bruce, V., \& Young, A. (1998). In the eye of the beholder. Oxford: Oxford University Press.

Carrasco, M., Penpeci-Talgar, C., \& Cameron, E. L. (2001). Characterizing visual performance fields: Effects of transient covert attention, spatial frequency, eccentricity, task and set size. Spatial Vision, 15, 61-75.

Chaiken, J. D., Corbin, H. H., \& Volkmann, J. (1962). Mapping a field of short-time visual search. Science, 138, 1327-1328.

Chambers, K. W., McBeath, M. K., Schiano, D. J., \& Metz, E. G. (1999). Tops are more salient than bottoms. Perception \& Psychophysics, 61(4), 625-635.

Chan, A. W., Kravitz, D. J., Truong, S., Arizpe, J., \& Baker, C. I. (2010). Cortical representations of bodies and faces are strongest in commonly experienced configurations. Nature Neuroscience, 13(4), $417-418$.

Christman, S. D. (1993). Local-global processing in the upper versus lower visual fields. Bulletin of the Psychonomic Society, 31(4), 275-278.

Coolican, J., Eskes, G. A., McMullen, P. A., \& Lecky, E. (2008). Perceptual biases in processing facial identity and emotion. Brain and Cognition, 66(2), 176-187.

Cumming, G., \& Finch, S. (2005). Inference by eye: Confidence intervals and how to read pictures of data. American Psychologist, 60(2), $170-180$.

Davenport, J. L. (2007). Consistency effects between objects in scenes. Memory \& Cognition, 35(3), 393-401.
Drain, M., \& Reuter-Lorenz, P. A. (1996). Vertical orienting control: evidence for attentional bias and "neglect" in the intact brain. Journal of Experimental Psychology: General, 125(2), 139-158.

Drummond, A., \& Tlauka, M. (2012). Vertical representational biases in healthy university students. Memory \& Cognition, 20(3), 210-216.

Edwards, M., \& Badcock, D. R. (1993). Asymmetries in the sensitivity to motion in depth: A centripetal bias. Perception, 22, 1013-1013.

Fecteau, J. H., Enns, J. T., \& Kingstone, A. (2000). Competition-Induced Visual Field Differences in Search. Psychological Science, 11(5), 386-393.

Felisberti, F. M., \& McDermott, M. R. (2013). Spatial location in brief, free-viewing face encoding modulates contextual face recognition. i-Perception, 4(5), 352-360.

Finkbeiner, M., Coltheart, M., \& Coltheart, V. (2014). Pointing the way to new constraints on the dynamical claims of computational models. Journal of Experimental Psychology: Human Perception and Performance, 40(1), 172-185.

Finkbeiner, M., \& Palermo, R. (2009). The role of spatial attention in nonconscious processing: A comparison of face and nonface stimuli. Psychological Science, 20(1), 42-51.

Fize, D., Cauchoix, M., \& Fabre-Thorpe, M. (2011). Humans and monkeys share visual representations. Proceedings of the National Academy of Sciences, 108(18), 7635-7640.

Fortenbaugh, F. C., Silver, M. A., \& Robertson, L. C. (2015). Individual differences in visual field shape modulate the effects of attention on the lower visual field advantage in crowding. Journal of Vision, 15(2), 19.

Haxby, J. V., Gobbini, M. I., Furey, M. L., Ishai, A., Schouten, J. L., \& Pietrini, P. (2001). Distributed and overlapping representations of faces and objects in ventral temporal cortex. Science, 293(5539), $2425-2430$.

Holmes, A., Kiss, M., \& Eimer, M. (2006). Attention modulates the processing of emotional expression triggered by foveal faces. Neuroscience Letters, 394(1), 48-52.

Holmes, A., Vuilleumier, P., \& Eimer, M. (2003). The processing of emotional facial expression is gated by spatial attention: evidence from event-related brain potentials. Cognitive Brain Research, 16(2), 174-184.

Hood, B. M., Willen, J. D., \& Driver, J. (1998). Adult's eyes trigger shifts of visual attention in human infants. Psychological Science, 9(2), 131-134.

Ishai, A., Ungerleider, L. G., Martin, A., Schouten, J. L., \& Haxby, J. V. (1999). Distributed representation of objects in the human ventral visual pathway. Proceedings of the National Academy of Sciences, 96(16), 9379-9384.

Karayanidis, F., Provost, A., Brown, S., Paton, B., \& Heathcote, A. (2011). Switch-specific and general preparation map onto different ERP components in a task-switching paradigm. Psychophysiology, 48(4), 559-568.

Kessler, K., \& Tipper, S. (2004). Retrieval of implicit inhibitory processes: The impact of visual field, object-identity, and memory dynamics. Visual Cognition, 11(8), 965-995.

Kliegl, R., Masson, M. E. J., \& Richter, E. M. (2010). A linear mixed model analysis of masked repetition priming. Visual Cognition, 18(5), 655-681.

Kok, P., Jehee, J. F., \& de Lange, F. P. (2012). Less is more: expectation sharpens representations in the primary visual cortex. Neuron, 75(2), 265-270.

Kravitz, D. J., Kriegeskorte, N., \& Baker, C. I. (2010). High-level visual object representations are constrained by position. Cerebral Cortex, 20(12), 2916-2925.

Kravitz, D. J., Saleem, K. S., Baker, C. I., Ungerleider, L. G., \& Mishkin, M. (2013). The ventral visual pathway: an expanded neural framework for the processing of object quality. Trends in Cognitive Sciences, 17(1), 26-49. 
Langton, S. R. H., Watt, R. J., \& Bruce, V. (2000). Do the eyes have it? Cues to the direction of social attention. Trends in Cognitive Sciences, 4(2), 50-59.

Levine, M. W., \& McAnany, J. J. (2005). The relative capabilities of the upper and lower visual hemifields. Vision Research, 45(21), 2820-2830.

Liu, L., \& Ioannides, A. A. (2010). Emotion separation is completed early and it depends on visual field presentation. PloS One, 5(3), e9790.

Martin, A., Wiggs, C. L., Ungerleider, L. G., \& Haxby, J. V. (1996). Neural correlates of category-specific knowledge. Nature, 379(6566), 649-652.

Masson, M. E. J., \& Kliegl, R. (2013). Modulation of additive and interactive effects in lexical decision by trial history. Journal of Experimental Psychology: Learning, Memory, and Cognition, 39(3), 898-914.

Naccache, L., Blandin, E., \& Dehaene, S. (2002). Unconscious masked priming depends on temporal attention. Psychological Science, 13(5), 416-424.

Niebauer, C. L., \& Christman, S. D. (1998). Upper and lower visual field differences in categorical and coordinate judgments. Psychonomic Bulletin \& Review, 5(1), 147-151.

Oldfield, R. C. (1971). The assessment and analysis of handedness: the Edinburgh inventory. Neuropsychologia, 9(1), 97-113.

Pessoa, L., McKenna, M., Gutierrez, E., \& Ungerleider, L. G. (2002). Neural processing of emotional faces requires attention. Proceedings of the National Academy of Sciences, 99(17), 11458-11463.

Previc, F. H. (1990). Functional specialization in the lower and upper visual fields in humans: Its ecological origins and neurophysiological implications. Behavioral and Brain Sciences, 13, 519-575.

Previc, F. H., \& Blume, J. L. (1993). Visual search asymmetries in threedimensional space. Vision Research, 33(18), 2697-2704.

Previc, F. H., \& Naegele, P. D. (2001). Target-tilt and vertical-hemifield asymmetries in free-scan search for 3-D targets. Perception \& Psychophysics, 63(3), 445-457.

Puri, A. M., \& Wojciulik, E. (2008). Expectation both helps and hinders object perception. Vision Research, 48(4), 589-597.

Quek, G. L., \& Finkbeiner, M. (2013). Spatial and temporal attention modulate the early stages of face processing: behavioural evidence from a reaching paradigm. PloS One, 8(2), e57365-e57365.

Quek, G. L., \& Finkbeiner, M. (2014a). Face-sex categorization is better above fixation than below: Evidence from the reach-to-touch paradigm. Cognitive, Affective, \& Behavioral Neuroscience, 14(4), 1407-1419.

Quek, G. L., \& Finkbeiner, M. (2014b). Gaining the upper hand: evidence of vertical asymmetry in sex-categorisation of human hands. Advances in Cognitive Psychology, 10(4), 131.

Reddy, L., Reddy, L., \& Koch, C. (2006). Face identification in the nearabsence of focal attention. Vision Research, 46, 2336-2343.
Reddy, L., Wilken, P., \& Koch, C. (2004). Face-gender discrimination is possible in the near-absence of attention. Journal of Vision, 4(2), $106-117$.

Rezec, A. A., \& Dobkins, K. R. (2004). Attentional weighting: a possible account of visual field asymmetries in visual search? Spatial Vision, 17(4-5), 269-293.

Rubin, N., Nakayama, K., \& Shapley, R. (1996). Enhanced perception of illusory contours in the lower versus upper visual hemifields. Science, 271, 651-652.

Schwarz, G. E. (1978). Estimating the dimension of a model. Annals of Statistics, 6(2), 461-464.

Sereno, M. I., Dale, A. M., Reppas, J. B., Kwong, K. K., Belliveau, J. W., Brady, T. J., . . Tootell, R. B. H. (1995). Borders of Multiple Visual Areas in Humans Revealed by Functional Magnetic Resonance Imaging. Science, 268(5212), 889-893.

Skrandies, W. (1987). The upper and lower visual field of man: Electrophysiological and functional differences. In D. Ottoson (Ed.), Progress in sensory physiology (pp. 1-93). Berlin: Springer.

Summerfield, C., \& Egner, T. (2009). Expectation (and attention) in visual cognition. Trends in Cognitive Sciences, 13(9), 403-409.

Talgar, C., \& Carrasco, M. (2002). Vertical meridian asymmetry in spatial resolution: Visual and attentional factors. Psychonomic Bulletin \& Review, 9(4), 714-722.

van Vugt, P., Fransen, I., Creten, W., \& Paquier, P. (2000). Line bisection performances of 650 normal children. Neuropsychologia, 38(6), 886-895.

Vuilleumier, P., Armony, J. L., Driver, J., \& Dolan, R. J. (2001). Effects of attention and emotion on face processing in the human brain: An event-related fMRI study. Neuron, 30(3), 829-841.

Wild, H. A., Barrett, S. E., Spence, M. J., O'Toole, A. J., Cheng, Y. D., \& Brooke, J. (2000). Recognition and sex categorisation of adults' and children's faces: Examining performance in the absence of sexstereotyped cues. Journal of Experimental Child Psychology, 77(4), 269-291.

Willenbockel, V., Sadr, J., Fiset, D., Horne, G., Gosselin, F., \& Tanaka, J. (2010). The SHINE toolbox for controlling low-level image properties. Journal of Vision, 10(7), 653.

Woestenburg, J. C., Verbaten, M. N., \& Slangen, J. L. (1983). The removal of the eye-movement artifact from the EEG by regression analysis in the frequency domain. Biological Psychology, 16(1-2), 127-147.

Wojciulik, E., Kanwisher, N., \& Driver, J. (1998). Covert visual attention modulates face-specific activity in the human fusiform gyrus: fMRI study. Journal of Neurophysiology, 79(3), 1574-1578.

Yund, E. W., Efron, R., \& Nichols, D. R. (1990). Detectability as a function of spatial location: effects of selective attention. Brain and Cognition, 12(1), 42-54.

Zeki, S. M. (1969). Representation of central visual fields in prestriate cortex of monkey. Brain Research, 14(2), 271-291. 